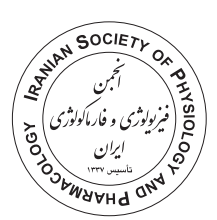

\title{
Royal jelly attenuated cognitive and non-cognitive deficits and blood-brain barrier disruption induced by ethidium bromide
}

\author{
${\text { Maryam Jamali }{ }^{1} \text {, Mahnaz Taherianfard }}^{1 *}$ (DD, Mahmood Aminlari², Jafar Jalaiee ${ }^{3}$
}

1. Physiology Division of Basic Science, School of Veterinary Medicine, Shiraz University, Shiraz, Iran

2. Biochemistry Divisions of Basic Science, School of Veterinary Medicine, Shiraz University, Shiraz, Iran

3. Pharmacology Divisions of Basic Science, School of Veterinary Medicine, Shiraz University, Shiraz, Iran

\begin{abstract}
Introduction: Multiple sclerosis (MS) is an autoimmune disease. The main aims of the present investigation were to evaluate the effect of royal jelly (RJ) on cognitive and noncognitive behavior, demyelination and the level of blood-brain barrier (BBB) disruption induced by ethidium bromide (EB).

Methods: Twenty-five adult male Sprague Dawley in five groups were used. Control (intact rat); Sham (surgery without EB injection); treatment control (EB injection without treatment); treatment1 and treatment2 (orally administered of RJ 100 and $200 \mathrm{mg} / \mathrm{kg} / \mathrm{day}$ after EB injection). EB ( $3 \mu 1$ of $0.01 \%$ ) injection in the dentate gyrus (DG) was used for demyelination. Demyelination induction was proved by histological examination. For the estimation of BBB integrity, Evans blue extravasation was done using an ELISA reader. Cognitive and non-cognitive behavior was evaluated by Morris water maze.

Results: Data showed that RJ corrected the deficit of demyelination. Cognitive and noncognitive behavior improved in treatment groups relative to treatment control by RJ. The extent of BBB disruption significantly improved in treatment groups compared to the treatment control group, in the whole brain and hippocampus.

Conclusion: Results indicate that RJ after EB injection in DG can improve cognitive and non-cognitive behavior, demyelination and BBB disruption in rat after EB injection. Therefore, it seems that RJ can be a supplementary drug for MS.
\end{abstract} Keywords: Royal jelly Morris water maze

Multiple sclerosis

\section{Introduction}

Multiple sclerosis (MS) is an autoimmune disease affecting over one million people worldwide (Tullman, 2013). Recent data indicated that traditional medicines could be impressive in MS disease therapy, by reducing demyelination (Mojaverrostami et al., 2018). Macroscopic examination of brain tissue in MS patients was revealed some deficits. The most deficiency is multiple sharply demarcated plaques in the white matter. Although, both white and gray matter

\footnotetext{
* Corresponding author: Mahnaz Taherianfard, taherian@shirazu.ac.ir

Received 27 July 2020; Revised from 10 March 2021; Accepted 15 March 2021
}

Citation: Jamali M, Taherianfard M, Aminlari M, Jalaiee J. Royal jelly attenuated cognitive and non-cognitive deficits and blood-brain barrier disruption induced by ethidium bromide. Physiology and Pharmacology 2021; 25: 353-362. http://dx.doi.org/10.52547/phypha.25.4.8 
destruction has been demonstrated (Mahad et al., 2008). Various mechanisms throughout the MS course, mainly regarding gray matter lesions and brain atrophy, lead to cognitive network dysfunction and can cause clinically significant cognitive impairment in roughly half the persons living with MS (Nasios et al., 2020). Hippocampal demyelination can disrupt the excitatory synapses and activation of neuronal signaling cascades, which modulate memory and learning (Dutta et al., 2011).

Direct injection of ethidium bromide (EB) is a simple tool for causing nerve cell destruction and helps researchers study the process of demyelination and remyelination (Bondan et al., 2000). This model easily can be used for studies of behavioral performance (Guazzo, 2005). The most critical stage in the MS disease is the blood-brain barrier (BBB) disruption. Evans blue dye (EBD) is an inert tracer that measures vascular permeability in animal models. In mammalian circulation, EBD is non-toxic and not metabolically active. In the recent two decades, the use of EBD to study BBB permeability has been increased in animals such as a rat (Saunders NR, 2015).

Royal jelly (RJ) contains proteins and phenols with high antioxidant concentration. Major royal jelly proteins exhibit antioxidant activities (Park et al., 2020). It is full of minerals and vitamins B, E, sex hormones and its gamma-globin strengthens the immune system. It is also useful for anorexia, stress and insomnia reduction (Barkhordari et al., 2013). It was showed that bee honey exerts a neuroprotective effect through its antioxidant activity (Ali and Kunugi, 2019). The prevention of muscle weakness is the most important therapeutic effect of RJ in MS patients (Oshvandi et al., 2020).

There are limited studies on the association between RJ, BBB penetration, demyelination in the hippocampus, cerebellum, corpus callosum (CC) and cognitive behavior after injection of EB. Therefore, the aims of the investigation were: 1 - the effect of RJ on the demyelination induce by EB in the hippocampus, cerebellum and CC; 2- the effect of RJ on cognitive and non-cognitive behavior disturbance after intra dentate gyrus (DG) injection of EB; 3- the effect of RJ on BBB penetration through Evans blue extravasation measurement in the brain and hippocampus after intra DG injection of EB.

\section{Materials and methods}

Procedures adopted in the present study were under Shiraz University ethical guidelines and compatible with the European Convention for the protection of vertebrate animals used for experimental and other scientific purposes (94GCU4M1755).

\section{Animals}

Twenty-five adult male Sprague Dawley rats were maintained under a light-dark cycle of $12 / 12 \mathrm{~h}$ and a temperature at $20 \pm 2^{\circ} \mathrm{C}$. Rats were access to free standard food and water. The animals were randomly divided into five groups. Control (intact rat); Sham (inter DG injection of $3 \mu 1$ normal saline); treatment control (inter DG injection of $3 \mu \mathrm{lEB}$ without treatment); experimental 1 and 2 (inter DG injection of $3 \mu 1 \mathrm{~EB}+$ two doses of $\mathrm{RJ})$. Induction of demyelination was done by injection of $3 \mu 1$ of $0.01 \%$ EB in DG, according to (Goudarzvand et al., 2016). RJ (SIGMA Aldrich, st. Louis, USA) was administered in two doses of 100 and $200 \mathrm{mg} / \mathrm{kg} /$ day from the 7th day after inter DG injection of $3 \mu 1 \mathrm{~EB}$ for 20 days by gavage (El-Nekeety et al., 2007).

\section{Stereotaxic procedure for induction of demyelination}

Animals anesthetized with an IP injection of ketamine $(100 \mathrm{mg} / \mathrm{kg})$ and xylazine $(10 \mathrm{mg} / \mathrm{kg})$. Rats fixed in the stereotaxic apparatus and EB were injected unilaterally into DG according to Paxinos and Watson atlas AP: $-4.9 \mathrm{~mm}$ relative to Bregma; $\mathrm{L}:+1.8 \mathrm{~mm}$ and DV: $6.9 \mathrm{~mm}$ under the skull.

The clinical scoring method for demyelination determination

Clinical symptoms of demyelination were observed on the 7th, 14th and 21st days after demyelination. The clinical grade was scored as follows: 0.5 , partial loss of tail tonicity; 1.0, complete loss of tail tonicity; 2.0, flaccid tail and abnormal gait; 3.0, hind leg paralysis; 4.0, hind leg paralysis with hind body paresis; 5.0, hind and foreleg paralysis and 6.0, moribund (Skundric et al., 2008).

\section{Morris water maze (MWM) apparatus}

The water maze used in the present study is a dark circular pool $(150 \mathrm{~cm}$ diameter and $60 \mathrm{~cm}$ height) filled with water to a depth of $45 \mathrm{~cm}$. A black metal platform $(10 \times 10 \mathrm{~cm})$ was located in the center of one of the 
arbitrarily designed north-east, south-east, south-west, or north-west orthogonal quadrants. The platform provided the only escape from the water. Four cues were housed on the walls of the water maze. The cues were kept fixed in positions during the study so that all of the rats could use the same visual cues. A CCD camera (Panasonic Inc., Japan) hanging from the ceiling above the MWM apparatus, recorded swimming and locomotion tracking was measured by a video tracking system for automated analysis of animal behavior using the neuro-vision software.

\section{MWM test for working memory}

The training sessions consisted of 4 trials per day (trial interval: 2min) for three consecutive days as visible platform sessions. The platform was visible with a flag and located in the center of one of the fourth quadrant and platform placed at a different quadrant each day. The rat must swim and find the platform for 90 s on the first day. If the rat cannot reach the platform within 90 s, it must direct to the platform by the experimenter. After finding the platform, rats remained on it for $60 \mathrm{~s}$. Hidden platform sessions consisted of four trials per day (trial interval: $2 \mathrm{~min}$ ), for three consecutive days. The platform was located in the center of one of the four quadrants. Each day the location of the platform was in a different quadrant. The time for finding the platform and rest on the platform was similar to the visible session. The probe test was given on day seven without a platform. However, in the neuro-vision program, the location of the platform was defined. For the recorded tracks, latency time and speed to reach target quadrants were calculated for subsequent analysis. In each training trial, cognitive performance was scored such that higher cognitive strategies received higher scores. The scaling was as follows: thigmotaxis $=1$, random $=2$, chaining $=$ 3 , focused $=4$, corrected $=5$ and direct $=6$.

\section{Evaluation of BBB permeability}

A $2 \%$ solution of Evans blue in normal saline $(4 \mathrm{ml} /$ $\mathrm{kg}$ body weight) was injected into the jugular vein. The stain was allowed to circulate for $0.5,3$ or $24 \mathrm{~h}$. In sham-operated animal, an intracardiac of Evans blue injection was done. The dye was allowed to circulate for $24 \mathrm{~h}$. Afterward, Rat transcardially perfused with $50 \mathrm{ml}$ of ice-cold PBS. Then the brain tissue removes and the hippocampus was isolated from the whole brain. The brain and hippocampus separately homogenized in $1.1 \mathrm{ml}$ of PBS sonicated and centrifuged (30min, 15000RPM, $\left.4^{\circ} \mathrm{C}\right)$. The supernatant was collected in aliquots. To every $0.5 \mathrm{ml}$ aliquot, an equal amount of $50 \%$ trichloroacetic acid was added, incubated overnight at $4^{\circ} \mathrm{C}$ and this was then centrifuged (30min, $\left.15000 \mathrm{RPM}, 4^{\circ} \mathrm{C}\right)$. Evans blue stain was measured by spectrophotometer (Thermo Spectronic Genesys 10 UV, Thermo Fischer Scientific Inc., Waltham, MA, USA) at $610 \mathrm{~nm}$ and quantified according to a standard curve. The results are presented as ( $\mu \mathrm{g}$ of Evans blue stain)/ ( $\mathrm{g}$ of tissue).

\section{Luxol fast blue staining for determination of Demyelination}

At the end of the experiment, for the histological study, rats were deeply anesthetized with a high dose of ketamine $(150 \mathrm{mg} / \mathrm{kg})$ based on bioethics. Then $20 \mathrm{ml}$ formaldehyde $4 \%$ as a fixative solution was perfused into the heart. After initial fixation, the animal's brain was carefully removed from the skull and tissue processing was used to prepare tissue block and then $5 \mu$ thick tissue sections by a microtome. Then Luxol fast blue staining was performed. To measurement of the myelination cross-section area imageJ software was used. The control group's brain structure cross-sectional area of myelination was considered $100 \%$ and another group was assessed relative to the control group.

\section{Statistical analysis}

For data analysis, SPSS (version 22) was used. The data analysis was done by one-way and repeated measure ANOVA; the Post-hoc test was Tuckey. A significant level was considered as $P<0.05$.

\section{Results}

\section{Effect of Rj on clinical score}

Figure 1 represents that 14 days after EB injection, the mean clinical severity score was significantly higher in the treatment control compared to both treatments groups $(P<0.01)$. Twenty-one days after EB injection, the mean clinical severity score was significantly lower in two treatment groups $(P<0.01)$ compared to the treatment control group and was significantly $(P<0.05)$ lower in treatment 2 relative to treatment 1 .

\section{Effect of RJ on the percentage of myelination}

Another way to prove demyelination after injection 


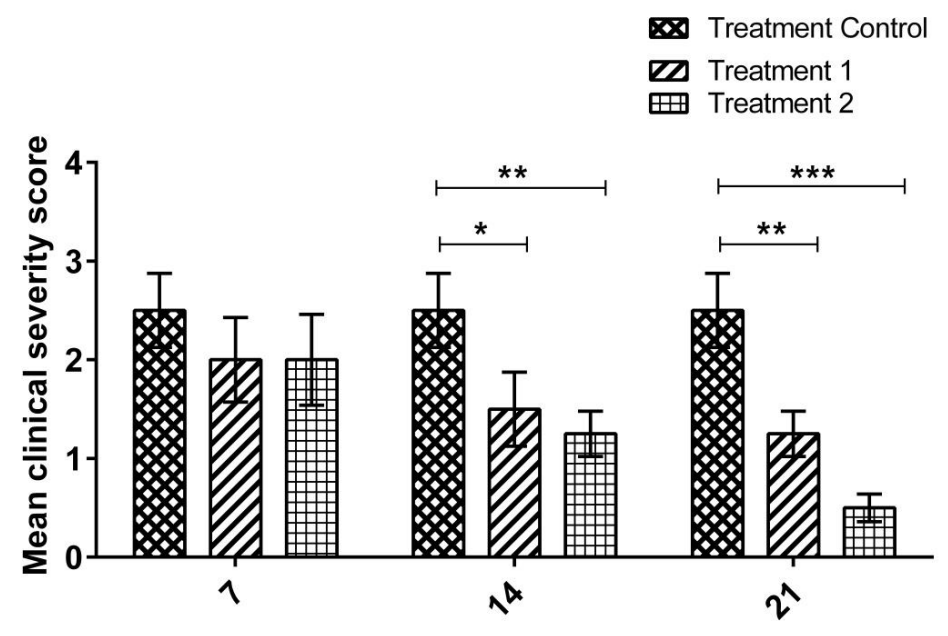

FIGURE 1. Effect of royal jelly on the mean clinical severity score in multiple sclerosis rats. ${ }^{*} P<0.05,{ }^{* *} P<0.01,{ }^{* * *} P<0.001$.

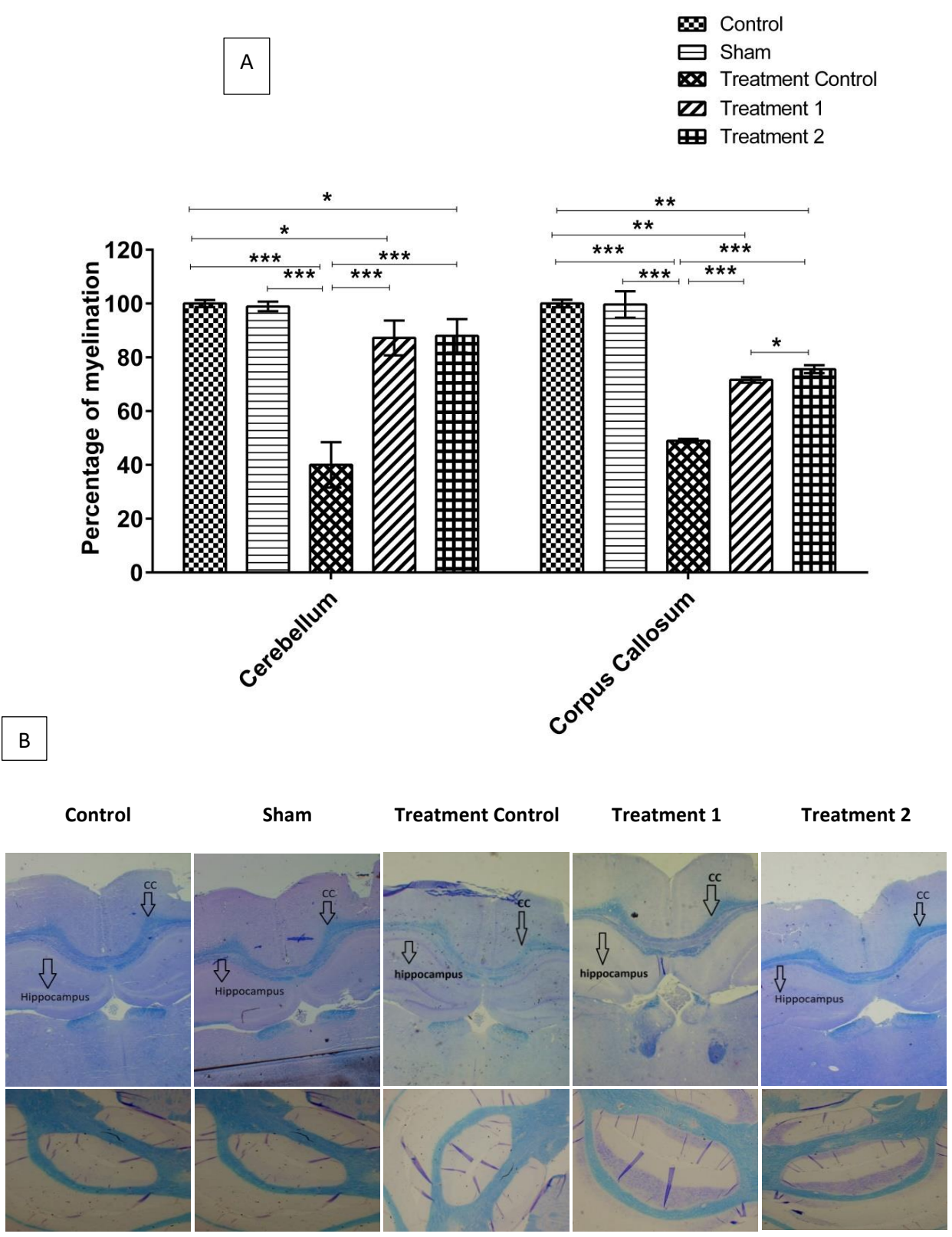

FIGURE 2. Effect of royal jelly on the hippocampus, corpus callosum (CC) and cerebellum. (A) The percentage of myelination; (B): photomicrograph of myelination in dentate gyrus, CC (B upper) and cerebellum (B lower) in all groups in ethidium bromide induced multiple sclerosis. ${ }^{*} P<0.05$ significance level relative to control and sham; ${ }^{* *} P<0.01 \mathrm{sig}$ nificance level relative to control and sham; ${ }^{* * *} P<0.001$ significance level relative to control and sham. 


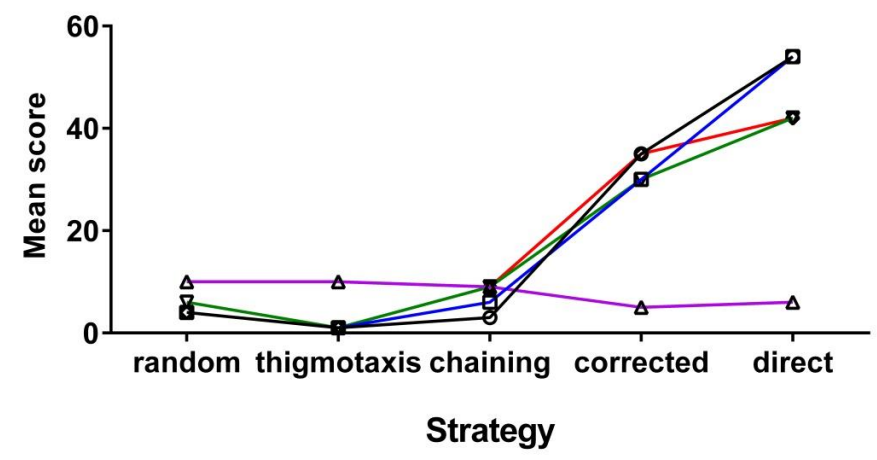

$\rightarrow$ control

\& sham

$\triangle$ Treatment Control

$\rightarrow$ Treatment 1

$\rightarrow$ Treatment 2

Strategy

FIGURE 3. Effect of royal jelly on the Morris water maze cognitive score in the probe test phase of multiple sclerosis rats.

$$
\text { A }
$$
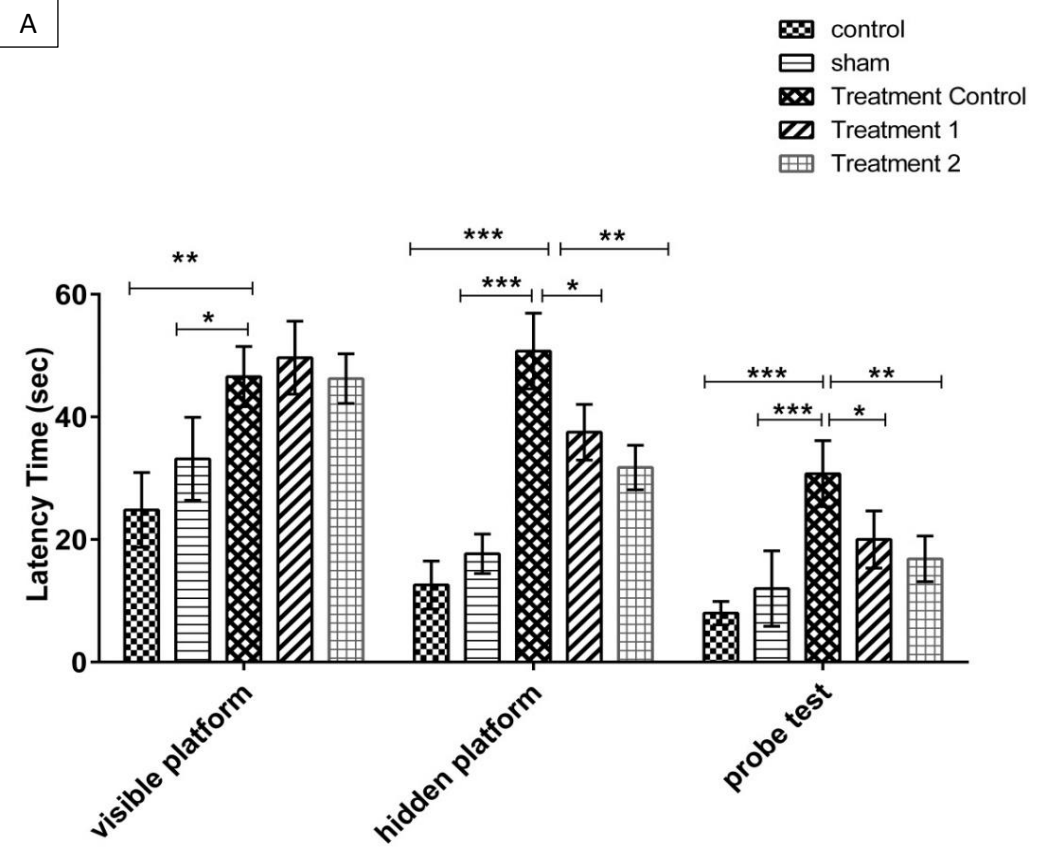

$\mathrm{B}$

$$
\begin{aligned}
& \text { control } \\
& \boxminus \text { sham } \\
& \text { Treatment Control } \\
& \text { ED Treatment } 1 \\
& \text { 四 Treatment } 2
\end{aligned}
$$

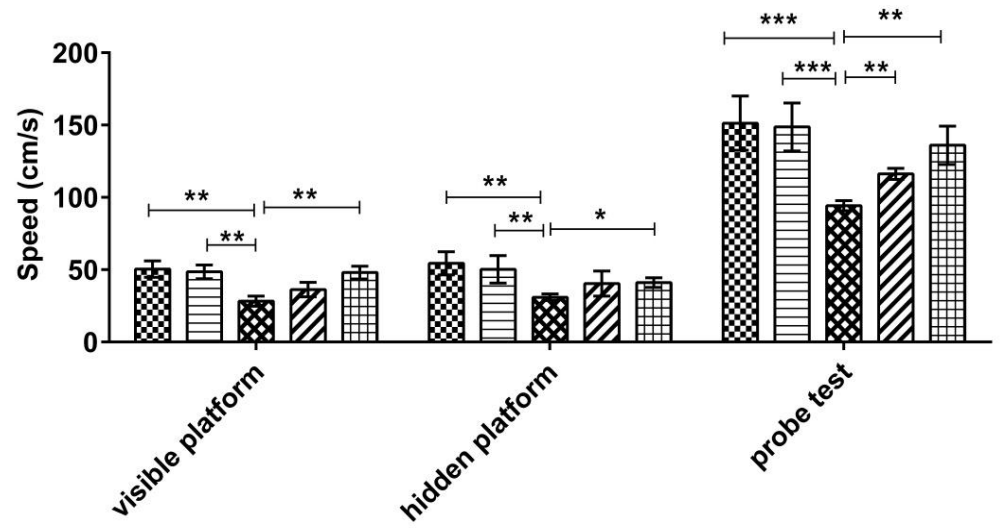

FIGURE 4. Effect of royal jelly on latency time (A) and speed (B) of the multiple sclerosis rats in the Morris water maze test. ${ }^{*} P<0.05$, ${ }^{* *} P<0.01,{ }^{* * *} P<0.001$. 


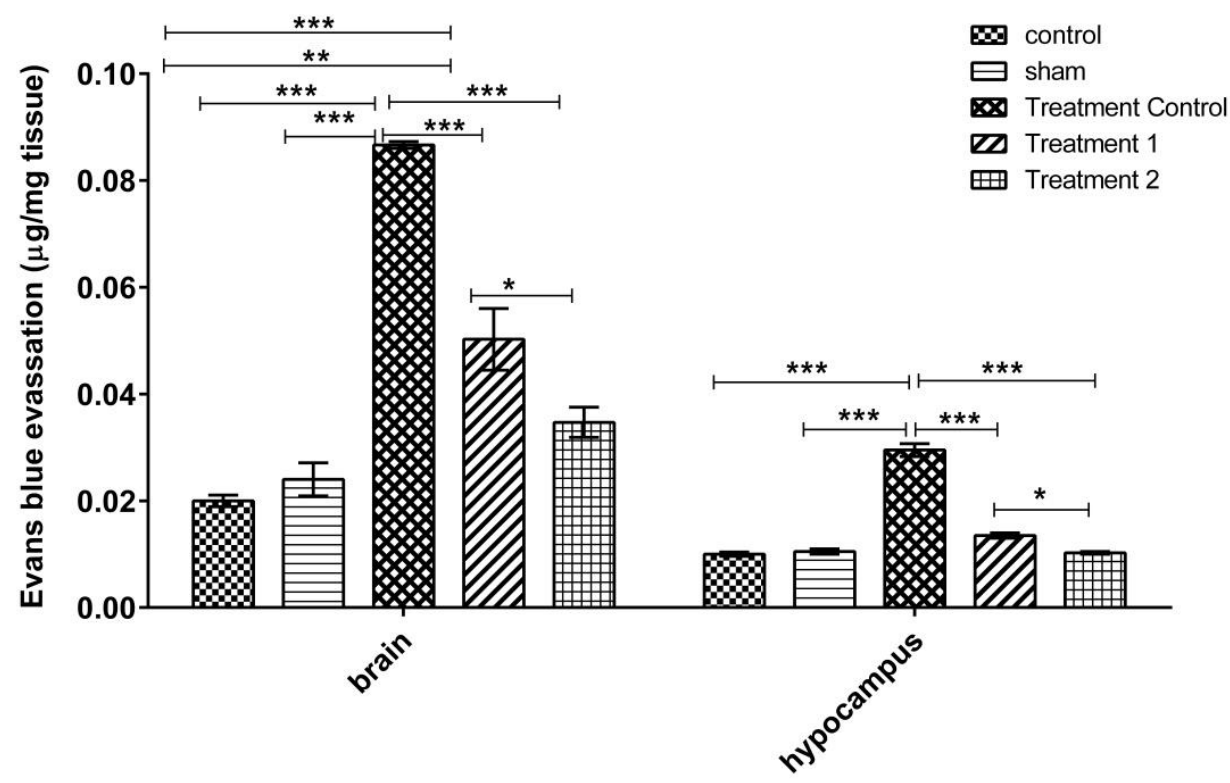

FIGURE 5. Effect of royal jelly on hippocampal and brain Evans blue extravasation in multiple sclerosis rats. ${ }^{*} P<0.05,{ }^{* *} P<0.01,{ }^{* * *} P<0.001$.

of EB was the determination of the percentage of the myelination in the hippocampus, corpus callosum and cerebellum. Figure 2A shows that the percent of the demyelination significantly reduced in the $\mathrm{CC}$ and cerebellum in treatment control $(P<0.001)$, treatment 1 $(P<0.001)$ and treatment $2(P<0.01)$ groups compared to the control group. However, both treatment groups had significantly higher myelination compared to treatment control. Treatment group 2 had significantly $(P<0.05)$ higher myelination than treatment group 1 in the hippocampus. In Figure 2B, the blue color in the photomicrograph represents the myelination and it confirms the percentage of myelination in Figure 2A.

\section{Effect of RJ on cognitive and non-cognitive behavior}

According to Figure 3 in the treatment control group, the thigmotaxis strategy was highest on the probe test day. However, in the other groups, corrected and direct strategies were the highest. The scores of these two strategies in both treatment groups were less than the control and sham groups. Figure 4A demonstrates that latency time invisible platform sessions was significantly higher in treatment control and both treatment groups compared to control $(P<0.001)$ and sham $(P<0.01)$ groups. In hidden platform sessions of the MWM test, latency time in the treatment control group was significantly higher than the control, sham $(P<0.001)$, treatment1 $(P<0.05)$ and treatment $2(P<0.01)$ groups. In both treatment groups, the latency time was significantly higher compared to control and sham $(P<0.01)$. In the probe test sessions, the results were similar to hidden platform sessions. However, the latency time was lower than the hidden platform sessions in all groups. Figure 4B shows that the treatment control group had significantly $(P<0.01)$ lower swimming speed than other groups, in the visible platform, hidden platform and probe test sessions of the MWM test.

\section{Effect of RJ on BBB permeability}

Evans blue extravasation represents the BBB disruption level. Figure 5 indicates that in treatment control groups, Evans blue extravasation was significantly $(P<0.001)$ higher than other groups in the brain and hippocampus. Brain Evans blue extravasation in both treatment groups was significantly $(P<0.01)$ higher than the control and sham groups. In the hippocampus, however, the treatment group 2 Evans blue extravasation was significantly $(P<0.05)$ lower than the treatment group 1 .

\section{Discussion}

In the present study, demyelination was performed by EB. Oligodendrocytes' death and destruction of BBB can cause by the EB administration, which leads to the production of reactive free radicals and impairment of cognitive and non-cognitive behavior as evaluated in the present by MWM test (Illouz et al., 2016). The results showed that during treatment, RJ at both doses reduced the severity of clinical symptoms. 
Present results showed that intra DG injection of EB led to the demyelination in the hippocampus, CC and cerebellum. On the other hand, RJ decreased demyelination and induced remyelination at both doses. DG is a brain structure that has been known to have a notable role in neurogenesis in many species of mammals, from rodents to primates (Cameron and McKay, 2001). DG is part of the hippocampal formation that had a gatekeeper role for the hippocampus (Dengler and Coulter, 2016). The hippocampus has many outputs to another part of the brain, but the most important output is the connection to some cortical structure such as the corpus callosum (Knezovic et al., 2019). On the other hand, the brain consists of two hemispheres, the cerebellum and the brainstem. The two hemispheres are separated by the longitudinal fissure across which there is a large connective band of fibers called the corpus callosum. The end part of the corpus callosum, towards the cerebellum, is called the splenium (Jonas and Lisman, 2015). Therefore, it seems that there are anatomical relationships between DG, CC as well as cerebellum and injury in one part can destroy other parts.

Various studies have shown that MS usually results from inflammation and oxidative stress in the CNS, damage to neurons, and demyelination, leading to varying degrees of clinical symptoms (Penkowa and Hidalgo, 2003). The RJ has antioxidant and anti-inflammatory properties. So, RJ increases the migration of oligodendrocyte precursor cells to the site of inflammation and reduces demyelination and subsequent clinical symptoms (Nagai et al., 2001; Kohno et al., 2004). Previous findings showed that RJ increases the differentiation of neurons, astrocytes and oligodendrocytes in the culture medium. Therefore in neurons and glial cells, RJ plays a role in the differentiation of stem cells, thereby inducing remyelination (Hattori et al., 2007). Hippocampal demyelination leads to cognitive deficits in intra DG injection of $\mathrm{EB}$ and remyelination can improve cognitive deficits. Previous studies suggested that RJ can facilitate neurogenesis and myelination in the hippocampal DG area (Hattori et al., 2011).

The most common cognitive deficits among MS patients include decreased information processing speed, memory loss and impaired spatial perception (Glanz et al., 2012). The MWM test examines cognitive and non-cognitive behavior and is a method of choice for spatial learning and memory investigation. In the MWM system, the visible and hidden platforms were related to learning and the probe test was related to memory (Choi et al., 2004). In this study, the MWM test was used to assess cognitive and non-cognitive behaviors. The index of cognitive behavior determination in the present study was thigmotaxis, chaining, corrected, random and direct swimming strategy. Non-cognitive behaviors investigate by latency time and swimming speed (Illouz et al., 2016). When animals are exposed to MWM for the first time, they usually exhibit a random swimming pattern to discover their surroundings and to have a more accurate picture of the space that they are swimming (Goodman and McIntyre, 2017). Few studies have been conducted on random behavior and most investigations are on thigmotaxis, corrected and direct behaviors.

Higaki et al. (2018) suggested that thigmotaxis is a swimming pattern associated with increased latency time and the percentage of this pattern indicates cognitive deficits in rodents. High cognitive strategies are direct and corrected in the MWM test, and changing the swimming strategy from thigmotaxis to direct and correct can be a demonstration of an increase in cognitive capacity, increasing efficiency over time, thereby reducing latency time during the steps of the MWM test (Illouz et al., 2016). The results showed that RJ on the probe test day led to an improvement in cognitive and non-cognitive behavior. This improvement is due to reduced latency to reach the platform, increased swimming speed, decreased thigmotaxis strategy and an increase in direct and corrected swimming in both treatment groups. However, both strategies of the direct and corrected in the treatment groups were significantly lower than both control and sham groups. Therefore, it is clear that $\mathrm{RJ}$ at doses used in this study might not just improve cognitive behavior, which is consistent with previous investigations (Tucker et al., 2018). Repeated activation of the afferent pathway to the hippocampus increases pyramidal cell responses. Consequently, it plays a role in hippocampal cell function and long-term potentiation of the hippocampus and improves learning and memory ability. On the other hand, RJ can affect intracellular signaling pathways. Therefore, RJ facilitates the recovery of cognitive behavior in the rat (Barak et al., 2013). In rats whose DG portion of the hippocampus was damaged by genetic manipulation of synaptic proteins, the probe phase showed lower direct and corrected swimming strategies. At this stage, rats also show 
an increase in latency time. These two behaviors suggest that the hippocampus is associated with cognitive impairment (Hattori et al., 2007).

In this study, the platform location were changed daily in the visible, hidden platforms and probe test. Thus, its impairments indicate a defect in spatial working memory in the rat model of MS, which agrees with previous studies (Rahimluy Marjani Z, 2014). Both treatment groups showed a significant decrease and the treatment control group showed a substantial increase in the latency to reach the platform compared to the control and sham groups in the visible and hidden platforms and probe test. Latency time in the MWM test is one of the main factors for learning and memory assessment, and its increase indicates impairment in spatial learning and memory. Thus, it is related to swimming strategy for cognitive deficits (Pyrzanowska et al., 2014).

For the representation of the BBB destruction, Evans blue extravasation was investigated. The results showed that MS increased BBB permeability in the hippocampus and another brain structure. Administration of RJ at both doses improved BBB permeability in the brain and hippocampus, but the improvement was incomplete. Seo et al. (2013) reported that reactive oxygen species and inflammatory mediators such as cytokines and chemokines increase BBB permeability. Free radicals attack unsaturated double-bonds of fatty acids in the plasma membrane of endothelial cells, cause damage and increased BBB permeability. A study reported that the $57 \mathrm{kDa}$ protein of RJ at a high dose of RJ activates the PKC, which leads to increase endothelial permeability and disruption of tight junctions of the paracellular epithelium (Kamakura and Fukushima, 2002). Myelin breakdown in MS dependent upon damage to the BBB and entry of inflammatory cells into the perivascular space and leading to the onset of clinical signs of the disease (Alder et al., 2000).

\section{Conclusion}

In the present study, RJ at two doses can have improvement effects by reducing $\mathrm{BBB}$ degradation, thereby decreasing clinical sign symptoms, increase axonal remyelination and modified spatial learning and memory in rats that received intra DG injection of EB. Therefore, RJ may be efficient as a pharmaceutical supplement in the treatment of MS.

\section{Conflict of interest}

None

\section{Acknowledgments}

This study was financially supported by Shiraz University (grant\#: 94GCU4M1755).

\section{References}

Ali AM, Kunugi H. Bee honey protects astrocytes against oxidative stress: a preliminary in vitro investigation. Neuropsychopharmacol Rep 2019; 39: 312-14. https://doi. org/10.1002/npr2.12079

Barak B, Okun E, Ben-Simon Y, Lavi A, Shapira R, Madar R, et al. Neuron-specific expression of tomosyn 1 in the mouse hippocampal dentate gyrus impairs spatial learning and memory. Neuromolecular Med 2013; 15: 351-63. https:// doi.org/10.1007/s12017-013-8223-4

Barkhordari F, Taavoni S, Haghani H, Gooshe Gir A. Effect of oral royal jelly on edema of premenstrual syndrome. Complement Med J 2013; 3: 355-65.

Bondan E, Lallo M, Sinhorini I, Pereira L, Graça D. The effect of cyclophosphamide on brainstem remyelination following local ethidium bromide injection in wistar rats. J Submicrosc Cytol Pathol 2000; 32: 603-12.

Cameron HA, McKay RD. Adult neurogenesis produces a large pool of new granule cells in the dentate gyrus. $\mathrm{J}$ Comp Neurol 2001; 435: 406-17. https://doi.org/10.1002/ cne. 1040

Choi JS, Park HJ, Jo YC, Chun MH, Chung JW, Kim JM, et al. Immunohistochemical localization of phospholipase $\mathrm{d} 2$ in embryonic rat brain. Neurosci Lett 2004; 357: 147-51. https://doi.org/10.1016/j.neulet.2003.12.054

Dengler CG, Coulter DA. Normal and epilepsy-associated pathologic function of the dentate gyrus. Prog Brain Res 2016; 226: 155-78. https://doi.org/10.1016/ bs.pbr.2016.04.005

Dutta R, Chang A, Doud MK, Kidd GJ, Ribaudo MV, Young EA, et al. Demyelination causes synaptic alterations in hippocampi from multiple sclerosis patients. Ann Neurol 2011; 69: 445-54. https://doi.org/10.1002/ana.22337

El-Nekeety AA, El-Kholy W, Abbas NF, Ebaid A, Amra HA, Abdel-Wahhab MA. Efficacy of royal jelly against the oxidative stress of fumonisin in rats. Toxicon 2007; 50: 25669. https://doi.org/10.1016/j.toxicon.2007.03.017

Glanz BI, Healy BC, Hviid LE, Chitnis T, Weiner HL. Cognitive deterioration in patients with early multiple sclerosis: A 5-year study. J Neurol Neurosurg Psychiatry 2012; 83: 
38-43. https://doi.org/10.1136/jnnp.2010.237834

Goodman J, McIntyre CK. Impaired spatial memory and enhanced habit memory in a rat model of post-traumatic stress disorder. Front Pharmacol 2017; 8: 663. https://doi. org/10.3389/fphar.2017.00663

Goudarzvand M, Choopani S, Shams A, Javan M, Khodaii Z, Ghamsari F, et al. Focal injection of ethidium bromide as a simple model to study cognitive deficit and its improvement. Basic Clin Neurosci 2016; 7: 63-72.

Guazzo EP. A technique for producing demyelination of the rat optic nerves. J Clin Neurosci 2005; 12: 54-8. https://doi.org/10.1016/j.jocn.2004.08.002

Hattori N, Nomoto H, Fukumitsu H, Mishima S, Furukawa S. Royal jelly-induced neurite outgrowth from rat pheochromocytoma pc12 cells requires integrin signal independent of activation of extracellular signal-regulated kinases. Biomed Res 2007; 28: 139-46. https://doi.org/10.2220/ biomedres.28.139

Hattori N, Ohta S, Sakamoto T, Mishima S, Furukawa S. Royal jelly facilitates restoration of the cognitive ability in trimethyltin-intoxicated mice. Evid Based Complement Alternat Med 2011; 2011: 165968. https://doi.org/10.1093/ ecam/nep029

Higaki A, Mogi M, Iwanami J, Min LJ, Bai HY, Shan BS, et al. Recognition of early stage thigmotaxis in morris water maze test with convolutional neural network. PLoS One 2018; 13: e0197003. https://doi.org/10.1371/journal. pone.0197003

Illouz T, Madar R, Clague C, Griffioen KJ, Louzoun Y, Okun E. Unbiased classification of spatial strategies in the barnes maze. Bioinformatics 2016; 32: 3314-20. https://doi. org/10.1093/bioinformatics/btw376

Jonas P, Lisman J. Structure, function, and plasticity of hippocampal dentate gyrus microcircuits: Frontiers, 2015. https:// doi.org/10.3389/978-2-88919-387-5

Kamakura M, Fukushima M. Inhibition of specific degradation of 57-kda protein in royal jelly during storage by ethylenediaminetetraacetic acid. Biosci Biotechnol Biochem 2002; 66: 175-78. https://doi.org/10.1271/bbb.66.175

Knezovic V, Kasprian G, Stajduhar A, Schwartz E, Weber M, Gruber GM, et al. Underdevelopment of the human hippocampus in callosal agenesis: an in vivo fetal mri study. AJNR Am J Neuroradiol 2019; 40: 576-81. https://doi. org/10.3174/ajnr.A5986

Kohno K, Okamoto I, Sano O, Arai N, Iwaki K, Ikeda M, et al. Royal jelly inhibits the production of proinflammatory cytokines by activated macrophages. Biosci Biotechnol Bio- chem 2004; 68: 138-45. https://doi.org/10.1271/bbb.68.138

Mahad D, Ziabreva I, Lassmann H, Turnbull D. Mitochondrial defects in acute multiple sclerosis lesions. Brain 2008; 131: 1722-35. https://doi.org/10.1093/brain/awn105

Mojaverrostami S, Bojnordi MN, Ghasemi-Kasman M, Ebrahimzadeh MA, Ghasemi Hamidabadi H. A review of herbal therapy in multiple sclerosis. Adv Pharm Bull 2018; 8: 57590. https://doi.org/10.15171/apb.2018.066

Nagai T, Sakai M, Inoue R, Inoue H, Suzuki N. Antioxidative activities of some commercially honeys, royal jelly, and propolis. Food chemistry 2001; 75: 237-40. https://doi. org/10.1016/S0308-8146(01)00193-5

Nasios G, Bakirtzis C, Messinis L. Cognitive impairment and brain reorganization in ms: underlying mechanisms and the role of neurorehabilitation. Front Neurol 2020; 11: 147. https://doi.org/10.3389/fneur.2020.00147

Oshvandi K, Aghamohammadi M, Kazemi F, Masoumi SZ, Mazdeh M, Vardanjani MM. Effect of royal jelly capsule on quality of life of patients with multiple sclerosis: a double-blind randomized controlled clinical trial. Iran Red Crescent Med J 2020; 22:e74.

Park MJ, Kim BY, Deng Y, Park HG, Choi YS, Lee KS, et al. Antioxidant capacity of major royal jelly proteins of honeybee (apis mellifera) royal jelly. J Asia Pac Entomol 2020; 23: 445-48. https://doi.org/10.1016/j.aspen.2020.03.007

Penkowa M, Hidalgo J. Treatment with metallothionein prevents demyelination and axonal damage and increases oligodendrocyte precursors and tissue repair during experimental autoimmune encephalomyelitis. J Neurosci Res 2003; 72: 574-86. https://doi.org/10.1002/jnr.10615

Pyrzanowska J, Piechal A, Blecharz-Klin K, Joniec-Maciejak I, Graikou K, Chinou I, et al. Long-term administration of greek royal jelly improves spatial memory and influences the concentration of brain neurotransmitters in naturally aged wistar male rats. J Ethnopharmacol 2014; 155: 343-51. https://doi.org/10.1016/j.jep.2014.05.032

Zahedeh Rahimluy M, Hatami H, AliHemmati AR. The investigation of the role of nitric oxide system in the spatial memory of rats in experimental model of multiple sclerosis. Razi J Med Sci 2014; 20: 49-58.

Saunders NR DK, Mollgard K and Habgood MD. Markers for blood-brain barrier integrity: how appropriate is evans blue in the twenty-first century and what are the alternatives? Front Neurosci. 2015; 9: 385. https://doi.org/10.3389/fnins.2015.00385

Seo JH, Miyamoto N, Hayakawa K, Pham LD, Maki T, Ayata $\mathrm{C}$, et al. Oligodendrocyte precursors induce early blood- 
brain barrier opening after white matter injury. J Clin Invest 2013; 123: 782-6. https://doi.org/10.1172/JCI65863

Skundric DS, Dai R, Zakarian VL, Zhou W. Autoimmune-induced preferential depletion of myelin-associated glycoprotein (mag) is genetically regulated in relapsing eae (b6 x sjl) f1 mice. Mol Neurodegener 2008; 3: 7. https://doi.org/10.1186/1750-1326-3-7
Tucker LB, Velosky AG, McCabe JT. Applications of the morris water maze in translational traumatic brain injury research. Neurosci Biobehav Rev 2018; 88: 187-200. https:// doi.org/10.1016/j.neubiorev.2018.03.010

Tullman MJ. A review of current and emerging therapeutic strategies in multiple sclerosis. Am J Manag Care 2013; 19: 21-7. 